\title{
[editorial]
}

\section{Factores pronósticos en nefritis lúpica}

\section{Paula Alba}

Profesor Asociado en Reumatología. Postgrado de Reumatología. Cátedra de Medicina I. Hospital Córdoba. Universidad Nacional de Córdoba.

El compromiso renal es reconocido como una de las complicaciones más serias en el LES, que ocurre a menudo dentro de los primeros cinco años de la enfermedad y es uno de los factores predictores de morbilidad y mortalidad. El pronóstico de pacientes con LES ha mejorado significativamente en las últimas décadas ${ }^{1,2,3}$. La sobrevida de pacientes con NL también se ha incrementado a más del $80 \%$ a los 5 años en la década del 90 comparado con el $50 \%$ previo reportado en los años $60^{4}$. La mejoría es particularmente marcada en los tipos proliferativos de glomerulonefritis y puede ser atribuido al reconocimiento y diagnóstico temprano así como al uso de terapias como los agentes citotóxicos y un mayor acceso a la diálisis y al trasplante ${ }^{5}$. Numerosos estudios epidemiológicos en pacientes con NL han sido publicados y más de trece factores de riesgo independientes predictores de progresión han sido identificados. Dentro de ellos se incluye la edad, sexo, raza, factor socioeconómico, polimorfismos genéticos, anticuerpos anti-ds-DNA, los anticuerpos antifosfolípidos (AAF), anticuerpos anti C1q, la clase histopatología, los índices de actividad (IA) y cronicidad (IC), la atrofia tubular, la trombosis capilar, la transformación histopatología, los niveles elevados de creatinina, el síndrome nefrótico, la hipertensión persistente, la falta de remisión clínica en el primer año, la hipocomplementemia, el retraso y el tipo de tratamiento instituido, los brotes renales y la falta de adherencia al tratamiento.

La etnicidad afroamericana, hispanoamericana y los mestizos latinoamericanos ${ }^{6}$ han sido identificados como factores de mal pronóstico renal con una sobrevida de $58 \%$ a los 5 años independiente del tratamiento. La presencia de ciertos polimorfismos genéticos podría explicar este resultado aunque el estado socioeconómico y la falta de la accesibilidad a servicios de salud son hechos que no pueden ignorarse en esta población. Un estudio triétnico en población americana ha demostrado recientemente que la pobreza es un factor importante en la progresión de las formas proliferativas ${ }^{7,8}$.

La edad y el sexo han sido identificados como factores no modificables. La edad más temprana a la presentación y el sexo masculino han sido descriptos como indicadores de mal pronóstico en series de adultos. Los brotes renales suelen ser precedidos de un aumento en los títulos de los anti-ds-DNA y predicen la ocurrencia de glomerulonefritis proliferativa. De esta manera, los anti-ds-DNA tienen un rol en el pronóstico renal por predisponer a formas más severas y brotes de la enfermedad'.

Los AAF han sido implicados como un factor pronóstico negativo en la sobrevida renal en 2 recientes estudios. Sin embargo el rol de éstos en el pronóstico de NL no ha sido claramente establecido ${ }^{10,11,12,13}$.

La relación entre los hallazgos histológicos y la evolución clínica de la NL han sido bien reconocidas. Los pacientes con glomerulonefritis mesangial (Clase II) y membranosa pura (Clase V) tienen generalmente mejor pronóstico renal así como un deterioro lento de la función renal en el tiempo. Por el contrario, las glomerulonefritis proliferativas (Clase III y IV) están asociadas a una evolución más agresiva con un deterioro de la función renal en la mayoría de los pacientes. Esta regla no puede ser tomada en cuenta en los subsiguientes brotes renales o en la transformación histológica que se pueda producir, ya que en estos casos el pronóstico depende de la nueva histología renal y la respuesta al tratamiento ${ }^{14}$.

Los IA e IC han sido indicadores de deterioro de la función renal en diferentes estudios pero estos están sujetos a diferencias ínter observador y tienen limitada reproducibilidad. Algunos estudios no han podido encontrar un claro punto de corte de los índices que sean clínicamente útiles para predecir fallo renal y mortali$\mathrm{dad}^{15}$. Debido a la fluctuación del proceso patológico en la NL y la potencial reversibilidad de algunas lesiones histológicas, los factores pronósticos pueden diferir dependiendo del tiempo de realización de la biopsia renal y del tratamiento realizado. Los hallazgos de laboratorio que predicen el pronóstico renal han sido extensamente estudiados. La elevación de la creatinina sérica y el síndrome nefrótico en la presentación de la NL, así como

\section{Correspondencia}

E-mail: paulaalba@yahoo.com 
la HTA persistente, el hematocrito bajo, la hipocomplementemia y el fracaso en lograr la remisión en el primer año del tratamiento son todos factores significativos ${ }^{15,16,17}$. Sin embargo, los pacientes con insuficiencia renal aguda pueden responder completamente al tratamiento y permanecer en remisión a largo plazo.

Los efectos de diferentes regímenes de tratamiento han influenciado el pronóstico en la NL. Sin embargo las controversias continúan con respecto a la duración de la terapia, diferentes regímenes y dosis.

En conclusión, el pronóstico renal está influenciado por múltiples factores en la NL en donde la etnicidad y nivel socioeconómico así como la presentación clínica, el perfil inmunológico, la histopatología y la respuesta inicial al tratamiento deben ser considerados.

\section{Bibliografía}

1. Stahl-Hallengren C, Jonsen A, Nived O, et al. Incidence studies of systemic lupus erythematosus in Southern Sweden: increasing age, decreasing frequency of renal manifestations and good prognosis. J Rheumatol 2000; 27:685-691.

2. Cervera R, Khamashta MA, Font J et al. Morbidity and mortality in systemic lupus erythematosus during a 5 year period. A multicenter prospective study of 1000 patients. European Working Party on Systemic Lupus Erythematosus. Medicine (Baltimore) 1999; 78:167-75.

3. Mok CC, Lee KW, Ho CT, Lau CS, Wong RW. A prospective study of survival and prognostic indicators of systemic lupus erythematosus in a southern chinese population. Rheumatology 2000; 39:399406.

4. Cameron JS. Lupus nephritis. J Am Soc Nephrol 1999; 10: 413-24.

5. Fiehn C, Hajjar Y, Nueller K, Waldherr R, Ho AD, Andrassy K. Improved clinical outcome of lupus nephritis during the past decade : importance of early diagnosis and treatment. Ann Rheum Dis 2003; 62: 435-39.

6. Pons Estel GJ, Alarcon GS, Burgos PI et al. Grupo Latinoamericano de Lupus GLADEL. Mestizos with systemic lupus erythematosus develope renal disease early while antimalarials retard its appearance: data from a Latin American Cohort. Lupus 2013; 22(9):899-907.
7. Alarcon GS, Mc Gwin GJr, Petri M, Reveille JD, Ramsey-Goldman R, Kimberly RP. PROFILE Study Group. Baseline characteristics of a multiethnic lupus cohort: PROFILE. Lupus 2002; 11:95-101.

8. Barr RG, Seliger S, Appel GB et al. Prognosis in proliferative lupus nephritis: the role of socioeconomic status and race/ethnicity. Nephrol Dial Transplant 18:2039-46; 2003.

9. Bootsma H, Spronk P, Derksen R, et al. Prevention of relapses in systemic lupus erythematosus. Lancet 1995; 345 (8965):1595-99.

10. Tektonidou M, Sotsiou F, Nakopoulou L, et al. Antiphospholipid Syndrome Nephropathy in patients with systemic lupus erythematosus and antiphospholipid antibodies. Arthritis Rheum 2004; 50:2569-79.

11. Bhandari S, Harnden P, Brownjohn AM, et al. Association of anticardiolipin antibodies with intraglomerular thrombi and renal dysfunction in lupus nephritis. QJM 1998; 91:401-9.

12. Moroni G, Ventura D, Riva P, Panzeri P et al. Antiphospholipid antibodies are associated with an increased risk for chronic renal insufficiency in patients with lupus nephritis. Am J of Kidney Diseases 2004; 43:28-36.

13. Sciascia S, Cuadrado MJ, Khamashta MA, Rocatello D. Renal Involvement in antiphospholipid syndrome. Nat Rev Nephrol 2014; 10(5):279-89.

14. Wang-Guo-Bao, Xu Zheng Jin, Liu Hong Fa et al. Changes in pathological pattern and treatment regimens based on repeat renal biopsy in lupus nephritis. Chin Med J 2012; 125:2890-94.

15. Schwartz MM, Lan SP, Bernstein J et al. Role of the pathology indices in the management of severe glomerulonephritis. LN Collaborative Study Group. Kidney Int 1992; 42:743-48.

16. Donadio JV, Hart GM, Bergstalh M, et al. Prognostic determinants in lupus nephritis: A long-term clinic-pathological study. Lupus 4: 109-115; 1995.

17. Hui M, Garner R, Rees F et al. Lupus nephritis a 15 year multi centre experience in the UK. Lupus 2013; 22:328-32. 